One of the likely consequences of Hubbell's and Bell's arguments is that there will probably be studies to resolve this issue in the near future. Theory must build on an understanding of simple cases and limiting assumptions, and Hubbell's deductions from 'neutral' assumptions have provided a rigorous basis for future work. At a time when books are often bland reviews of papers published many years before, this book has a high proportion of previously unpublished results and stimulating ideas. It is unlikely to be ignored.

Peter A. Abrams is in the Department of Zoology,

University of Toronto, 25 Harbord Street,

Toronto, Ontario M5S 3G5, Canada.

\section{Fertility from a metabolic viewpoint}

\section{On Fertile Ground: A Natural History of Human Reproduction by Peter T. Ellison \\ Harvard University Press, 2001. 358 pp.}

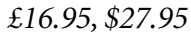

\section{Roger V. Short}

Peter Ellison is Professor of Anthropology and Dean of the Graduate School of Arts and Sciences at Harvard. He is best known for his exploitation of methods for measuring reproductive hormones in human saliva, thereby making it possible to carry out non-invasive studies of the reproductive endocrinology of people living in remote parts of the world, such as the Ituri rainforest in Central Africa or the mountains of Nepal. This book therefore describes some of his work on the fertility of traditional human societies.

Unfortunately, On Fertile Ground: A Natural History of Human Reproduction does not live up to its grandiose title; it would have been better called "Females, fatness and fertility", as this is its central theme. The opening chapters present a superficial (and sometimes incorrect) account of human reproduction, with strained analogies: "the area at the base of the brain called the hypothalamus and an associated bit of glandular tissue called the pituitary gland that hangs off the base of the brain like a tiny holiday ornament." It is excessively and unnecessarily North American in its outlook and citation of references. For example, pages are devoted to the bizarre and discredited views of Margie Profet, who postulated that menstruation was designed to cleanse a woman's reproductive tract of pathogens. Although Ellison cites references in the bibliography to important articles that completely demolish Profet's hypothesis, he never presents these counter-arguments in the text.

Another major failing of the book is the almost complete lack of figures or diagrams to leaven a turgid text. There are repeated references to the important sex differences in the shape of the human pelvis: "the pubic bones at the front of the pelvis are proportionately larger in females, giving the top of the true pelvis, or pelvic inlet, a more circular and less heart-shaped outline, while the ischial bones and the sacrum at the bottom of the true pelvis, or pelvic outlet, have spread further apart, increasing the diameter of the outlet and making it more circular in form as well." Oh for an illustration! And it would have been interesting to have had some discussion about when and how this sexual dimorphism has developed.

Ellison devotes a major section of the book to breastfeeding. Unfortunately, there is no mention of the entero-mammary circulation, which supplies specific immunoglobulins in breast milk to counteract enteric infections in the baby. As diarrhoea is the single greatest killer of babies worldwide, its prevention is a vital piece of our natural history. There is no mention of the inhibitory peptide that accumulates in the alveoli of the mammary gland and turns off milk production if the milk is not removed, thereby making the mammary gland a unique supply-meets-demand organ. There is no mention of the fact that human nipple sensitivity increases greatly on the day of birth, or that in animals, each suckling bout sends afferent neural inputs to the hypothalamus, resulting in the central release of $\beta$-endorphin, the 'feel-good hormone', that depresses dopamine secretion and hence elevates prolactin.

Likewise, there is no mention of the fact that in animals, denervation of the teat immediately abolishes the inhibitory effects of lactation on ovarian activity. Instead, Ellison chooses to dismiss the results of P. W. Howie and A. S. McNeilly, who showed in the 1980s that suckling frequency and duration are unquestionably related to the duration of lactational amenorrhoea. In its place, he proposes a "strong alternative hypothesis,"

Fertility symbol: fatness and fecundity have long been linked in the human imagination. in which "relative metabolic load" is said to be responsible for the lactational inhibition of ovulation. Although we are given no clues as to how the body might sense this "relative metabolic load," or how this information is then used to inhibit ovulation, we are assured that his hypothesis fits the available data better than the nursing frequency hypothesis, and is sounder theoretically! How come that high-yielding dairy cows in negative energy balance, which are milked only twice a day, show little inhibition of ovulation postpartum, whereas lowyielding beef cattle, frequently suckling their calves, have a far longer period of lactational anovulation?

There is a good rebuttal of Rose Frisch's hypothesis that the percentage of body fat is the trigger for the onset of female puberty. But Ellison then goes on to propose an alternative "pelvic size hypothesis," suggesting (without any evidence) that there are mechanical determinants of the timing of puberty. Curiously, there is no mention of the timing of the onset of spermatogenesis in boys, or how it is that boys grow to be considerably taller than girls.

Indeed, the male gets short shrift throughout this book. We are told, quite incorrectly, that follicle-stimulating-hormone in the male is sometimes referred to as interstitial cell-stimulating hormone, that Sertoli cells are also called interstitial cells, that the location of the gametogenic cells is the first anatomical distinction between an ovary and a testis and that "sperm counts above 15,000 or 20,000 per millilitre of semen are considered 'normal'"' And I was amazed to learn that "the thermal oscillations produced in the mucin strands by the woman's body heat also resonate with the beating of the sperm cell's tail to aid its progress," although no reference is given to the source of this incredible information.

Sadly, this book is not the place I would recommend anybody to go for a comprehensive and factually correct account of the "natural history of human reproduction".

Roger V. Short is in the Department of Obstetrics and Gynaecology, University of Melbourne, 132 Grattan Street, Melbourne 3053, Australia. 